\title{
Behavioral Modeling Techniques for Teaching Communication Circuits and Systems
}

\author{
José M. de la Rosa \\ Instituto de Microelectrónica de Sevilla, IMSE-CNM (CSIC/Universidad de Sevilla) \\ C/Américo Vespucio, 41092 Sevilla, SPAIN \\ E-mail: jrosa@imse-cnm.csic.es
}

\begin{abstract}
This paper discusses the use of behavioral simulation techniques to improve the quality of teaching/learning circuits and systems for communications. The proposed pedagogical methodology has been applied in several electrical engineering courses, in both undergraduate and master degrees. The method allows students to better understand some complex circuitand physical-level phenomena, by describing them at a higher abstraction level. In addition to enhance their understanding of design problems and skills, students become more motivated and satisfied. As an application, two case studies are considered in this work: a radio-frequency front-end system and an analog-todigital converter. In both cases, behavioral models of the different building blocks have been implemented in MATLAB/SIMULINK and used by the students enrolled in two courses named: Electronic Circuits for Communications and Wireless Transceivers: Standards, Techniques and Architectures ${ }^{1}$.
\end{abstract}

\section{INTRODUCTION}

Simulation is an essential tool for the analysis and verification of integrated circuits. In the case of analog circuits, SPICE-like simulators have been traditionally used to evaluate their performance at different stages of the design procedure. However, the increasing complexity of analog systems - for instance in telecom applications - yield excessively long simulation CPU times. To cope with this problem, different alternative simulation techniques have been developed which improve the computational efficiency at the price of reducing the accuracy in their circuit-element models. Among others, one of the best accuracy-speed trade-offs is achieved by the so-called behavioral simulation approach. In this approach, a given circuit is partitioned into a set of sub-circuits - often called building blocks - modeled by a set of equations referred to as behavioral laws or behavioral models. This way, the accuracy of the simulation depends on how precisely these behavioral models emulate their actual operation [1].

From an educational perspective, the benefits of teaching behavioral modeling and simulation are twofold. On the one hand, students learn practical skills, which are essential for their professional career. On the other hand, they get a better insight about design problems in complex circuits and systems, because they deal with design parameters which are much

\footnotetext{
${ }^{1}$ This work was supported in part by the Spanish Ministry of Science and Innovation (with support from the European Regional Development Fund) under contract TEC2010-14825/MIC, in part by the Consejería de Innovación, Ciencia y Empresa, Junta de Andalucía, under contract TIC-2532, and in part by the I Plan Propio de Docencia de la Universidad de Sevilla, ELECOM project.
}

closer to the system-level specifications than transistor sizes and biasing, whose impact on the overall performance metrics is sometimes quite difficult to understand [2].

In this scenario, this work proposes the use of behavioral models as an effective pedagogical tool to enhance the quality of teaching and learning in electrical engineering, and particularly in those courses focused on analog circuits intended for communications. Two case studies are considered. The first one shows how to learn the system planning process of Radio Frequency (RF) front-ends in wireless transceivers. The second one focuses on how to teach students to design Analog-to-Digital Converters (ADCs) by means of behavioral simulation. In both cases, behavioral models are implemented in MATLAB/SIMULINK, thus benefiting from its friendly user interface, powerful signal processing capability and high flexibility to incorporate new models. The teaching strategies presented in this paper - based on the experiences the author have had in the last ten years while teaching both undergraduate and master degree courses - demonstrate that the proposed simulation-based educational method help electrical engineering students comprehend the course material, being an efficient complement to traditional lectures presentations and lab exercises.

\section{Importance of Learning Simulation Techniques}

In addition to teach the fundamental principles of operation and practical design issues, course contents dealing with circuits and systems must be supported by an appropriate systematic design procedure, in which students can put the lessons learnt into practice. One of the most common approaches is based on the well-known top-down/bottom-up hierarchical synthesis methodology. In this approach, a system is divided into several abstraction levels, so that at each level, a design or sizing process takes place, by transmitting (or mapping) the system specifications in a hierarchical way - from the top level to the bottom level. The reverse path is followed to implement the hierarchical bottom-up verification process [3].

Different simulation approaches and their corresponding design parameters may be needed at each abstraction level of the system hierarchy in order to optimize the trade-off between simulation CPU time and the accuracy of results. Therefore, learning the different modeling and simulation approaches is a must for those students enrolled in electrical engineering courses. 
The simulation-based learning techniques carried out in this work are intended for telecom circuits and systems. To this end, three different hierarchy levels are defined: system level (transceiver), building-block level (low-noise amplifiers, mixers, oscillators, analog-to-digital converters, etc.), and circuit level (operational amplifiers, switches, capacitors, etc.). The fundamental operation of all these entities, as well as the main circuit effect causing a deviation from their ideal operation, are described as behavioral models implemented in the MATLAB/SIMULINK environment. This well-known mathematical package - which constitutes a standard CAD platform today in science and engineering - presents a number of advantages for students in terms of friendliness of the user interface; flexibility for the extension of new elements and a huge number of tools for signal post-processing [4].

Different model implementation approaches have been followed: one is based on the interconnection of SIMULINK standard library blocks [4]. The resulting block diagrams are very intuitive for students to evaluate communication systems with simple models, at the price of increasing the CPU time and reducing the accuracy. The second modeling approach consists of using the so-called SIMULINK S-functions, which allows to simulate more complex circuit and physical-level phenomena in an efficient way, although they are not so intuitive for students and require to learn some basic skills on computer languages such as $\mathrm{C}$ [4].

\section{COURSE DESCRIPTIONS}

The presented simulation-based educational methodology has been implemented in the following courses dealing with circuits and systems for communications:

- The first one, named Electronic Circuits for Communications $(E C)$, is an undergraduate course given in two different bachelor programs at the University of Seville: an Electrical and Electronic Engineering degree and a Telecomunications Engineering degree. In both cases, the subjects covered in the course are focused on the design of analog, mixed-signal and RF integrated circuits for communications, putting emphasis on the design issues at building-block level.

- The second one, named Wireless Transceivers: Standards, Techniques and Architectures (WT), belongs to a master degree in Microelectronics, and covers different topics mainly focused on the transceiver architecture level of wireless communication systems.

Both courses are given in one semester, organized as a 15week class period, and correspond to six European Credit Transfer System (ECTS) credits, three of them being theoretical ECTS credits and the other three ones corresponding to practical ECTS credits. The contents of both courses are summarized below.

\section{A. EC Undergraduate Course}

The EC course aims at teaching fundamental principles and practical design issues dealing with integrated circuits included in the analog front-ends of main telecom systems. The operation of these circuits is analysed considering that they are part of a more complex system. Therefore, it is important to study not only their stand-alone operation but also the impact of their circuit non-ideal effects on the performance of entire telecom systems. From this point of view, the use of behavioral modeling and simulation techniques constitute a very powerful tool to study the propagation of error mechanisms through the different hierarchical levels of the system.

Bearing this general learning objective in mind, the contents of the course are structured around three main blocks:

- Part I - Introduction and General Design Considerations: this part of the course comprises two units that give a panoramic overview of circuits and systems used in telecom systems, and explain their basic concepts and design issues like noise figure, linearity, low power and low-voltage operation.

- Part II - Analog/Digital Interfaces, which includes three units covering fundamentals and architectures of analogto-digital and digital-to-analog converters for telecom systems.

- Part III - Signal Conditioning Circuits: this part, made up of three units, explains the main design issues and circuit techniques to implement amplifiers, filters, mixers, oscillators and frequency synthesizers required to implement the analog front-end of digital communication systems.

\section{B. WT Master Course}

The objective of the WT master course is to study the main wireless communication systems, putting especial emphasis on those important issues related to the design of Radio Frequency (RF) CMOS Integrated Circuits, from the perspective of wireless transceivers. The fulfillment of this general objective is itemized in the following partial objectives:

- To analyze and to compare different architectures of digital radio transceivers.

- To study the main wireless communication standards and their application to the design of integrated circuits for communications.

- To learn the most important figures of merit and specifications required to characterize the performance of RF transceivers.

- To learn practical issues related to design methodologies and tools used to design and to synthesize RF transceivers.

The contents of WT master course are linked to the aforementioned objectives, and are organized in three parts:

- Part I - Introduction: this part includes one unit that gives an overview of wireless communication systems, standards, trends and challenges.

- Part II - Signal Processing Techniques and Wireless Communications: this part comprises two units. The first one deals with codification and modulation techniques, and the second one focuses on multiple access techniques and communication standards. 
- Part III - RF Transceivers: Architectures and Design Considerations: this part is divided into two units and explains the most important transceiver architectures as well as practical issues related to the system planning and design methodology.

\section{Learning Methodology and CASE Studies}

The courses described in previous section are taught by combining classical learning materials - like lecture notes, slides and practical/lab exercises - with multimedia resources and e-learning modules. The latter are integrated in the WebCT virtual learning environment [5].

All these learning contents are complemented with case studies. This allows students to put theory into practice in an easy way thanks to the use of the behavioral model of different communication systems in SIMULINK. To this end, every academic year, at least one case study is linked to the second and the third part of the courses described above. This section gives a description of two cases studies. The first one linked to Part II of the EC undergraduate course - consists of the high-level design of $\Sigma \Delta$ Modulators ( $\Sigma \Delta \mathrm{Ms}$ ) for telecom applications. The second case study - given in the Part III of the WT master course - deals with the system planning and simulation of Direct Conversion Receivers (DCRs).

\section{A. Case Study 1: Modeling and Simulation of $\Sigma \Delta M s$}

The EC course includes several case studies that aim to teach students how to use behavioral modeling and simulation techniques to analyze the effect of circuit errors on the performance of ADCs, in order to map system-level specifications onto building-block specifications in a high-level sizing process. To this purpose, different approaches for the implementation of behavioral models of both pipeline [6] and $\Sigma \Delta$ ADCs [7], [8] have been considered in the course.

As an illustration, Fig. 1 shows the block diagram of a 2ndorder $\Sigma \Delta \mathrm{M}$ implemented using behavioral models based on S-functions as proposed in [8]. In this case study, behavioral modeling techniques are used with a twofold objective. On the one hand, students learn fundamental concepts of ADCs and how to obtain their main performance metrics like output spectra, harmonic distortion, Signal-to-Noise Ratio (SNR), etc. On the other hand, a number of routines and MATLAB scripts are available in the toolbox used during the course. Thanks to the use of behavioral models, students can easily understand the principle of operation of basic circuits as well as the influence of their most important limiting factors, like thermal noise, finite dc gain, Gain BandWidth (GBW) product, SlewRate (SR), etc. Moreover, they can learn how to model these non-ideal effects in order to analyze complex circuits and systems used in communications, studying both their isolate effect as well as their cumulative effect.

\section{B. Case Study 2: Modeling and Simulation of DCRs}

The main purpose of this case study is to learn how to analyze and to simulate wireless transceivers at the system level, but taking into consideration circuit-level effects. To this

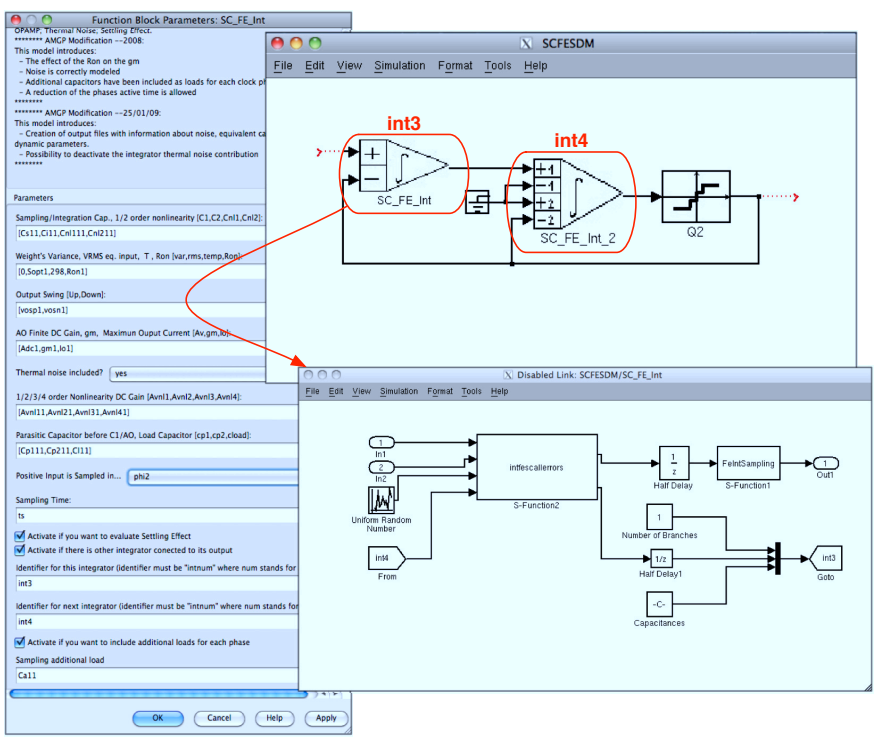

Fig. 1. Illustrating some behavioral models used to simulate $\Sigma \Delta \mathrm{Ms}$.

purpose, a DCR is simulated in SIMULINK environment using the behavioral model available in the SIMULINK block set presented in [9]. This block set includes a SIMULINK library with the main RF circuit models that are needed to implement wireless transceivers, namely: low-noise amplifiers, mixers, oscillators, filters and programmable amplifiers. There is also a library including other building blocks like the antenna, the duplexer filter and RF switches, which are also required to implement reconfigurable architectures.

Behavioral models of building blocks include the main ideal functionality as well as the following non-idealities: thermal noise (characterized by the Noise Figure (NF) and SNR), and non-linearity - represented by the input-referred second- and third-order intercept points. In addition to these general parameters, some block-specific errors have also been included, like oscillator phase noise and mixer offset [9]. As an illustration, Fig. 2 shows the implementation of the DCR in SIMULINK, highlighting some parts of the behavioral models.

In order to help students with the use of the mentioned SIMULINK block set, several MATLAB scripts are available in the WebCT site of the course. Some of these scripts are

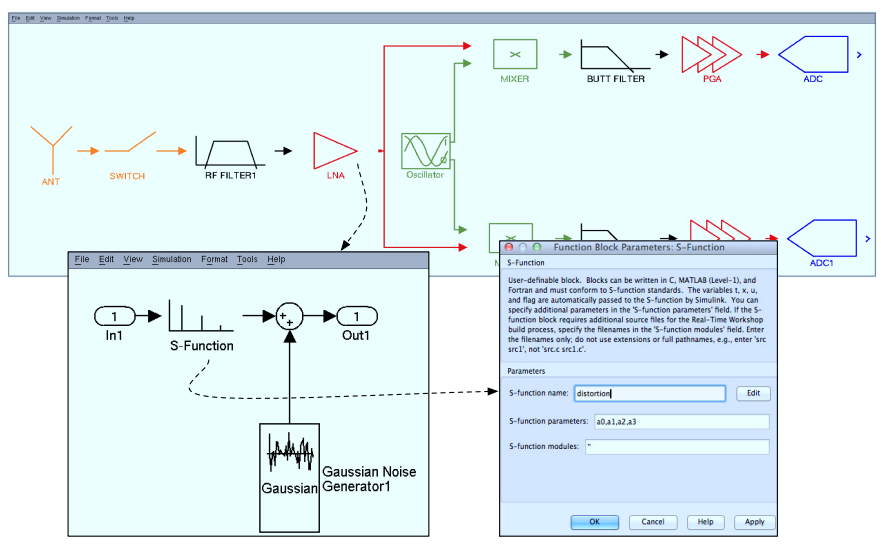

Fig. 2. Illustrating some behavioral models used to simulate DCRs. 
used to set up receiver parameters, like gain, noise figure, intermodulation distortion intercept points, etc. Other scripts are used to simulate performance metrics like NF, output spectra and level diagrams. This way, the explanation of the behavioral models and MATLAB scripts in classroom, provides students with complementary skills that may be quite useful for them in their professional career, since they may follow similar approaches to model other complex systems.

\section{Results and Students' Feedback}

Students attending EC course were surveyed during four consecutive academic years in order to express their opinion and give us their feedback to improve the quality of the course in terms of its contents, learning resources and the pedagogic procedure. The number of students participating in this survey varied every year, being an average of 50 participants approximately.

Fig. 3 shows the interest and motivation demonstrated by the students for those course contents taught by using the proposed simulation-based methodology. Note that a huge majority of students appreciated the use of behavioral simulation as a learning resource, increasing year after year (except for the third year surveyed) and more than $90 \%$ of them become interested in the proposed methodology in the last year.

Students were asked about their level of satisfaction with the simulation-based methodology, assigning a grade from 1 to 4 , with 1 being "bad" and 4 being "very good". As illustrated in Fig. 4, students were roughly well satisfied, especially in the last years, when the proposed simulated-based methodology became more mature and refined. Indeed, the highest level of satisfaction was obtained in the last year, when more than 50\% of students expressed the maximum level of satisfaction.

Finally, Fig. 5 shows the overall impression given by students. It is clear that a vast majority of them had a good impression of the course, becoming better in the last two years, and particularly in the last one, when almost all students considered the course either "good" $(\sim 70 \%)$ or "very good" $(\sim 30 \%)$.

\section{CONCLUSION}

The use of behavioral modeling and simulation techniques has been presented as a teaching methodology used in different courses dealing with circuits and systems for wireless telecom

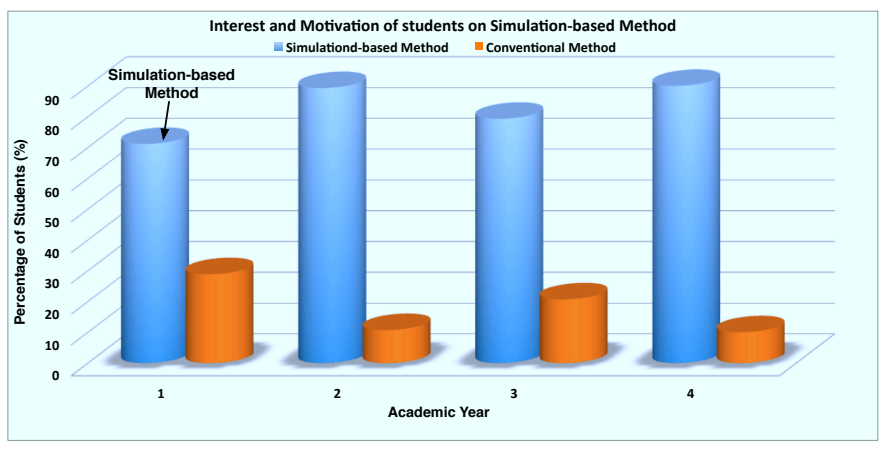

Fig. 3. Interest and motivation of students.

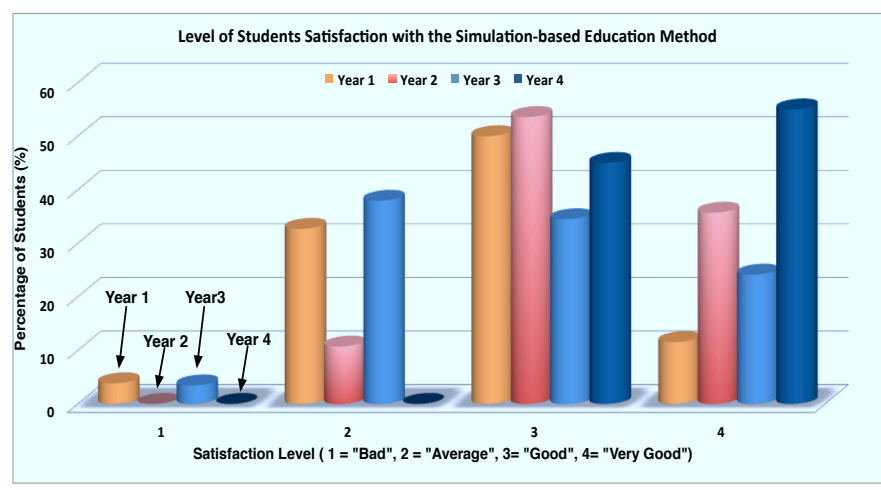

Fig. 4. Students' feedback on the proposed method.

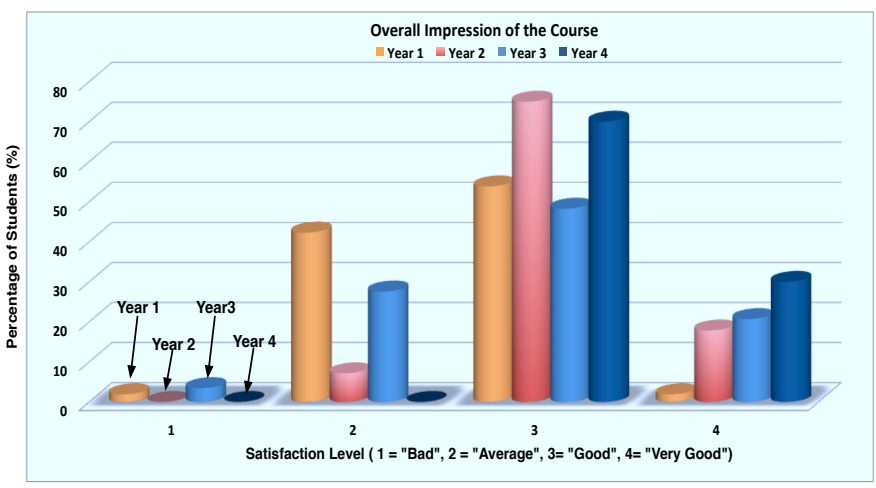

Fig. 5. Overall impression of the course given by students.

applications. Based on more than ten years experience, it has been demonstrated to be a method well appreciated by the majority of students, who prefer the course contents including simulation-based learning resources to those taught using classical methods.

\section{REFERENCES}

[1] A. Stefan et al., "Behavioral Models for Microwave Circuit Optimization," Proc. of IEEE Global Engineering Education Conference (EDUCON), pp. 806-808, 2011.

[2] A. Abramovitz, "Teaching Behavioral Modeling and Simulation Techniques for Power Electronics Courses," IEEE Trans. on Education, Accepted for publication.

[3] G. Gielen and J. Franca, "CAD Tools for Data Converter Design: An Overview," IEEE Trans. on Circuits and Systems II: Analog and Digital Signal Processing, vol. 43, pp. 77-89, February 1996.

[4] Mathworks, MATLAB R2011b Documentation. [Online]. Available: http://www.mathworks.com, 2011.

[5] D. Pishva et al., "A Survey on How Blackboard is Assisting Educational Institutions Around the World and the Future Trends," Proc. of IEEE Int. Conference on Advanced Communication Technology (ICACT), pp. 1539$1543,2010$.

[6] J. Ruiz-Amaya et al., "Behavioral Modeling Simulation and High-Level Synthesis of Pipeline A/D Converters," Proc. of IEEE Int. Symposium on Circuits and Systems (ISCAS), pp. 5609-5612, 2005.

[7] P. Malcovati et al., "Behavioral modeling of switched-capacitor sigmadelta modulators," IEEE Trans. on Circuits and Systems - I: Regular Papers, vol. 50, pp. 352-364, March 2003.

[8] J. Ruiz-Amaya et al., "High-Level Synthesis of Switched-Capacitor, Switched-Current and Continuous-Time $\Sigma \Delta$ Modulators Using SIMULINK-based Time-Domain Behavioral Models," IEEE Trans. on Circuits and Systems - I: Regular Papers, vol. 51, pp. 1795-1810, September 2005.

[9] A. Morgado et al., "A SMULINK Block Set for the High-Level Simulation of Multistandard Radio Receivers," Proc. of IEEE Int. Symposium on Circuits and Systems (ISCAS), pp. 2950-2953, 2007. 